\title{
Fabrication of hydroxyapatite/PLA composite nanofiber by electrospinning
}

\author{
K. Tanaka, T. Shiga \& T. Katayama \\ Department of Biomedical Engineering, Doshisha University, Japan
}

\begin{abstract}
In recent years, bone tissue engineering has been getting attention. The basic elements of bone tissue engineering include the following: an appropriate cell source, optimal culture conditions and a biodegradable scaffold. The scaffold which is worked as an extra cellular matrix to organize cells into a threedimensional structure is used as a space filling material. Nanofibers are expected to be used as the scaffold because their small sizes are close to the structural dimension of the extra cellular matrix of native tissues and organs. Thermoplastic polymers have been spun into nanofibers by the electrospinning method that applies a high voltage to polymer solution. Among many thermoplastic polymers, PLA is expected to be used for the scaffold because of its biocompatibility, bioabsorbability and mechanical properties. To obtain better affinity properties of PLA nanofiber scaffold with the bone, compounding of hydroxyapatite (HAp) nanoparticles that have the same composition formula with the human bone has been conducted and cell culture properties have been evaluated. It is known that HAp form clusters in the polymer solution because of their high surface activity, and the clusters cause reduction of nanofiber strength. Surface modification of HAp is needed to disperse HAp in the nanofiber. Since the mechanical characteristics of the scaffold are important for providing sufficient temporary mechanical support to withstand in vitro stresses, it is necessary to evaluate the mechanical property. In this study, HAp/PLA composite nanofibers are fabricated by electrospinning, and their mechanical properties are evaluated by tensile tests of nonwoven fabrics. HAp/PLA nanofiber nonwoven fabric showed lower tensile strength compared to PLA nanofiber nonwoven fabrics. By dispersing HAp uniformly in the nanofibers, HAp/PLA nanofiber nonwoven fabrics tended to show high strength.

Keywords: nanofiber, poly-lactic acid (PLA), hydroxyapatite, mechanical property, electrospinning, nonwoven fabrics.
\end{abstract}




\section{Introduction}

In recent years, bone regeneration for various bone diseases such as bone infections, bone tumors and bone loss by trauma has been expected [1]. The therapies for bone defects, autografts, allografts, xenografts and other artificial substitutes have been conducted, however, these substitutes have their specific problems and limitations [1]. Recent research has been attracting attentions for bone tissue engineering by combining artificial materials with specific living cells. The basic elements of bone tissue engineering include the following: an appropriate cell source, optimal culture conditions and a biodegradable scaffold [2]. The scaffold (polymer, collagen, ceramics, etc.) which is worked as an extra cellular matrix to organize cells into three-dimensional structures is used as a space filling materials [3]. In addition to the cell culture property, the mechanical characteristics of the scaffold for bone tissue engineering are important for providing sufficient mechanical support to withstand in vitro stresses [4]. Among many polymers to be used for the scaffold, PLA is expected to be used for bone tissue engineering because of its good biocompatibility, bioabsorbability and tough mechanical properties. To enhance affinity with the bone, hydroxyapatite (HAp) that has the same composition formula with the human bone has been applied as the composite material with PLA. Although HAp/PLA composite materials had been established, it was difficult to produce a suitable structure for the scaffold that increases cell adhesion and regenerative potential [5]. Nanofiber structure is suited to the scaffold because its nanometer size is close to the structural dimension of the extra cellular matrix of native tissues and organs [6]. Since nanofibers have some special characteristics, such as nanometer sized diameter, high surface area to volume ratio, and good alignment of molecular chain, the cell adhesion and proliferation are more enhanced than with macrostructure or microstructure scaffolds $[1,5]$. Thermoplastic polymers have been spun into nanofibers by the electrospinning method that applies a high voltage to polymer solution. In previous studies, HAp composite nanofibers (HAp/PGA, HAp/PCL, HAp/PLA, etc.) have been fabricated by electrospinning for the scaffold. Because of the high surface activity of HAp nanoparticles, they are reported to form clusters in the polymer solution [7]. Since the clusters in the nanofibers caused the strength reduction, it is important to disperse HAp uniformly in the nanofibers. Hot water treatment and surface modification by surfactant (oleic acid, stearic acid, etc.) are expected for the dispersion processes of HAp. To fabricate high strength scaffold for bone tissue engineering, it is necessary to clarify the effect of dispersion of HAp on the mechanical properties. In this study, hot water treatment, oleic acid treatment and stearic acid treatment were used to disperse HAp in the nanofibers. HAp/PLA composite nanofibers were fabricated by the electrospinning process and the mechanical properties of HAp/PLA nanofiber nonwoven fabrics were evaluated by tensile test. 


\section{Dispersion of HAp in nanofibers}

\subsection{Material and preparation of polymer solution}

PLA (poly-lactic acid, Mw: 18000, Zhejiang Hisun Biomaterials) is used for the material of nanofibers. Hydroxyapatite $\left(\mathrm{HAp}, \mathrm{Ca}_{10}\left(\mathrm{PO}_{4}\right)_{3}(\mathrm{OH})_{2}, 80 \mathrm{~nm}\right.$ in diameter, $500 \mathrm{~nm}$ in length, Wako Chemicals) is used for the particles to be added to PLA. Dichloromethane (DCM) was used as the dissolution solvent, and dimethyl-formamide (DMF) was used to enhance conductivity of the solution (DCM: $\mathrm{DMF}=8: 2$ ). The polymer solution was prepared by adding PLA pellets (10 $\mathrm{wt} \%$ for solution) and HAp (25 wt \% for PLA) to DCM, and it was stirred at $30^{\circ} \mathrm{C}$ for 60 minutes. Then, DMF was added to the solution and stirred at $30{ }^{\circ} \mathrm{C}$ for 5 minutes.

For dispersion of HAp, hot water treatment and surface treatment by the surfactants were carried out. Hot water treatment in which HAp is kept in hot water $\left(100^{\circ} \mathrm{C}\right)$ for 48 hours is the method that absorbed hydroxyls to nanoparticles. Surfactant was expected to absorb on HAp and it improve the wettability of HAp. In this study, oleic acid and hydroxyl stearic acid were used as the surfactants $\left(10^{2} \mathrm{~mol} / \mathrm{dm}^{3}\right.$ for solution).

\subsection{Fabrication of PLA/HAp composite nanofibers}

The nanofibers were fabricated by the electrospinning unit (NANON, MECC). Figure 1 shows a schematic diagram of the electrospinning process. Polymer solution is ejected as a fine jet of liquid from the tip of the needle and is deposited on the collector by applying a high voltage to the polymer solution. Nanofibers were fabricated at room temperature $\left(25^{\circ} \mathrm{C}\right)$ and $30 \pm 5 \%$ of relative humidity. The high voltage between the needle tip and the target was set to $16 \mathrm{kV}$. Nanofibers were spun to aluminum foil at $150 \mathrm{~mm}$ distance on a drum collector (Fig. 2, $50 \mathrm{rpm}$ rotation speed). The feed speed of the syringe was set at $0.2 \times 10^{-1} \mathrm{~mm} / \mathrm{min}$. Electrospun nanofibers were observed by the scanning electron microscope (SEM JSM-6390LT, JOEL Ltd., Japan)

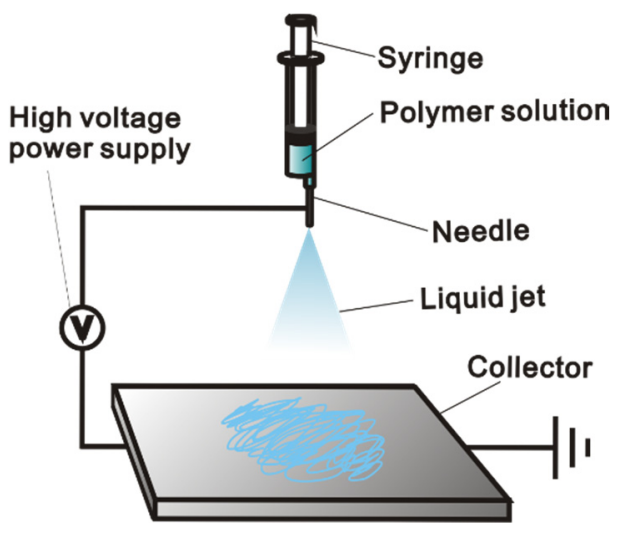

Figure 1: Schematic diagram of the electrospinning process. 
PLA nanofibers and four types of HAp/PLA composite nanofibers that are called "untreated", "hot water treated" that used hot water treated HAp, "oleic acid treated" that used oleic acid treated HAp and "stearic acid treated" that used stearic acid treated HAp were fabricated by electrospinning.

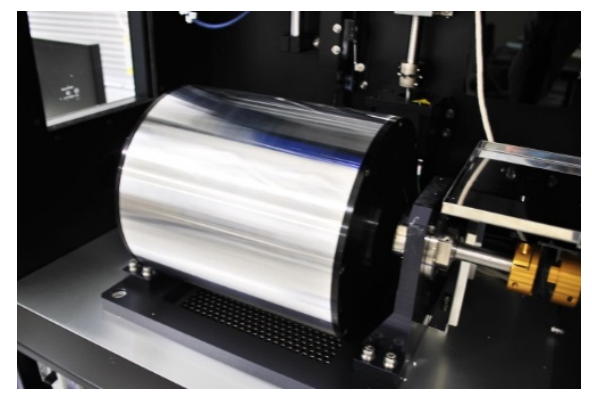

Figure 2: Drum collector.

\subsection{Result and discussion}

Figure 3 shows SEM images of the fabricated HAp/PLA composite nanofibers. For Untreated, HAp particles were clustered in the nanofibers. For hot water treated and oleic acid treated, HAp particles were dispersed than untreated. For stearic acid treated, HAp particles were mostly dispersed in the nanofiber. Figure 4 shows the histogram of cluster size of HAp particles. While high

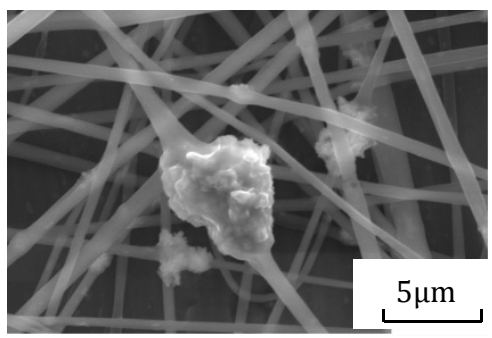

(a) Untreated

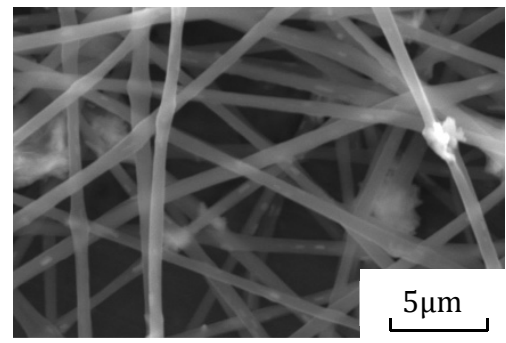

(c) Oleic acid treated

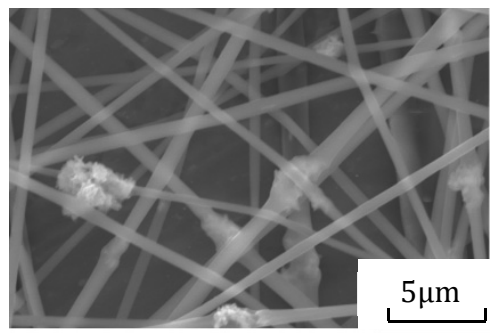

(b) Hot water treated

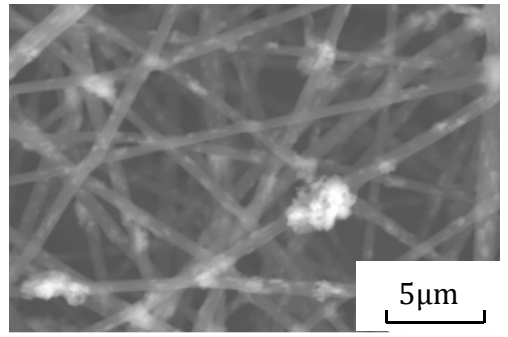

(d) Stearic acid treated

Figure 3: SEM image of HAp/PLA composite nanofibers. 
frequency can be observed in a big cluster size for the histogram of untreated, high frequency can be observed in small cluster size for the histogram of stearic acid treated. These results indicated that the stearic acid treatment can disperse HAp in the nanofibers. Figure 5 shows the possible dispersing mechanism of stearic acid treatment. These results were considered to be cause by the improvement of wettability of HAp due to hydrophilic and lipophilic groups which stearic acid has [9]. Stearic acid is physically absorbed with HAp, and it tends to wet in the organic solvent. In addition, when the molecular chains were worked as the steric barrier, and the dispersed condition was kept [9].

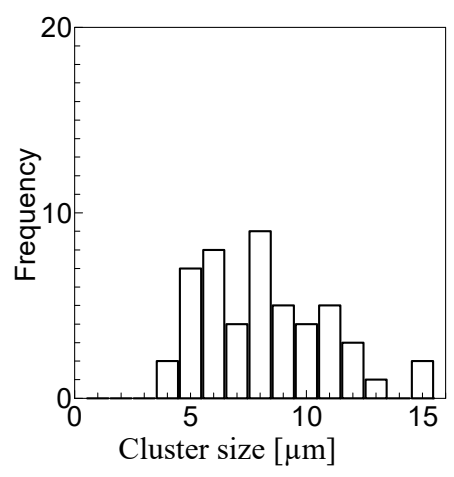

(a) Untreated

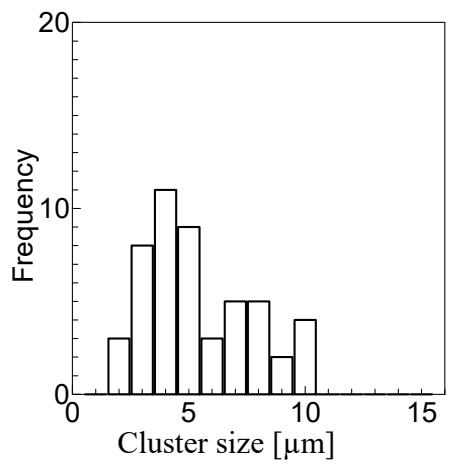

(c) Oleic acid treated

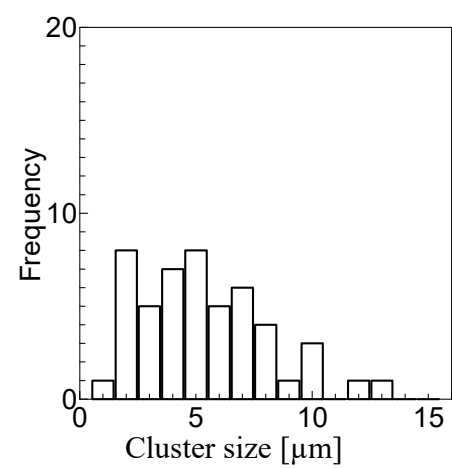

(b) Hot water treated

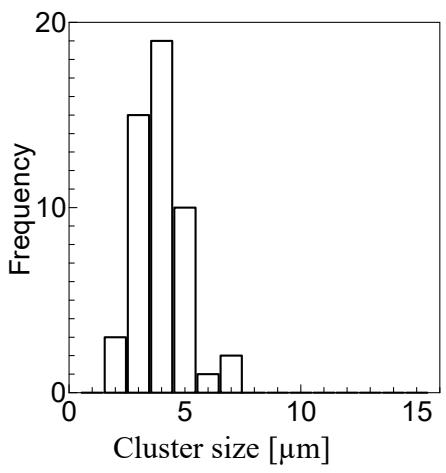

(d) Stearic acid treated

Figure 4: Histogram of cluster size. 


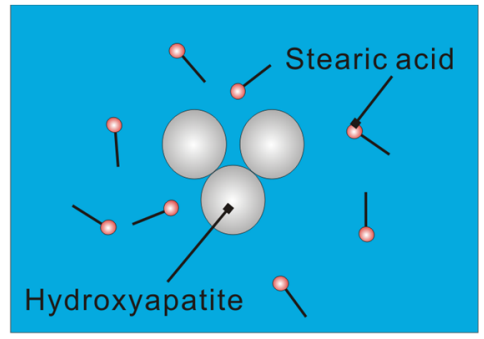

(a) Aggregation

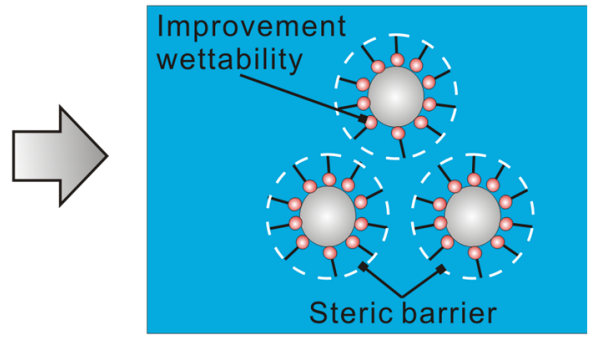

(b) Deflocculation

Figure 5: Dispersion mechanisms.

\section{Evaluation of tensile properties of HAp/PLA nanofiber nonwoven fabrics}

\subsection{Materials and method}

The following three types of nanofiber nonwoven fabrics were used: PLA nanofibers, untreated HAp/PLA composite nanofibers and stearic acid treated HAp/PLA composite nanofibers, which showed the highest dispersed condition in section 2.4. Figure 6 shows the photo of the tensile test specimen. As shown in Figure 7, the tensile test specimens were prepared by punching the nanofiber nonwoven fabric with a sealing frame of polyester film and it was glued to another frame.

The cross section of the nanofiber nonwoven fabrics which was used for calculation of stress was calculated by the thickness (measured with micro meter) and the width (measured with vernier caliper) of the tensile test specimen. Tensile tests were conducted at a displacement rate of $1 \mathrm{~mm} / \mathrm{min}$ by using the universal testing machine (EZ-graph, Shimadzu Co., Japan) equipped with $5 \mathrm{~N}$ load cells.

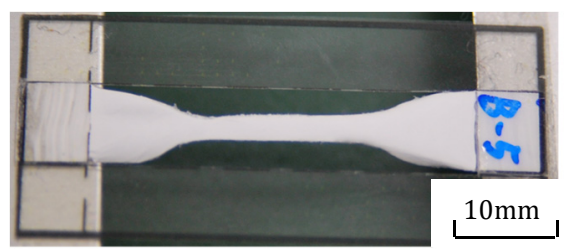

Figure 6: Tensile test specimen. 


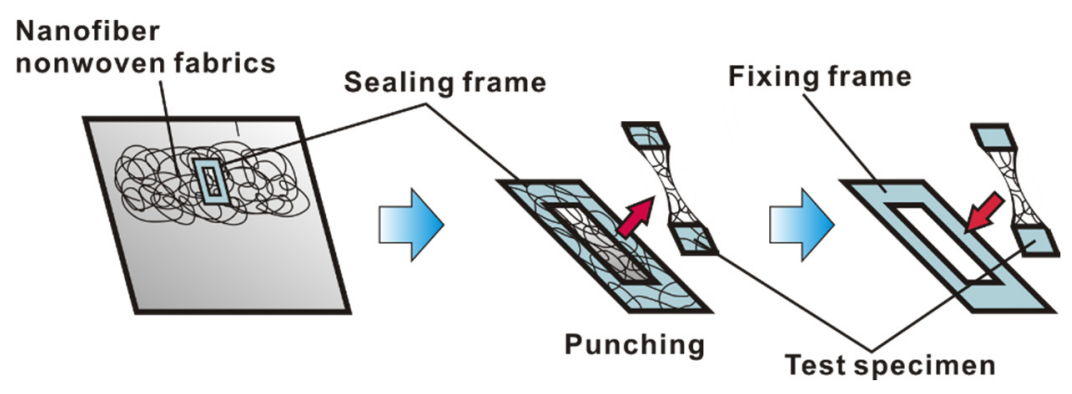

Figure 7: Procedure of making tensile test specimen.

\subsection{Result and discussion}

Figure 8 shows the stress-strain curves and tensile strength of three types of nanofiber nonwoven fabrics. While PLA nanofiber has the strength of $4.5 \pm 0.5 \mathrm{MPa}$, untreated HAp/PLA composite nanofiber has only $1.0 \pm 0.2 \mathrm{MPa}$, and stearic acid treated HAp/PLA composite nanofiber has $1.5 \pm 0.1 \mathrm{MPa}$. The result shows that the combination with HAp caused the decrease in fiber strength. Previous studies suggested that the aggregation of nanoparticles caused the stress concentration. Stress concentration acted as the starting point of fiber cracks. Stearic acid treated HAp/PLA composite nanofibers tended to show high strength. It is suggested that stress concentration was suppressed by dispersion of HAp. However, it is much lower than the PLA nanofibers strength. This fracture characteristic is considered to be due to weak interfacial adhesion between PLA and HAp [10]. Interfacial adhesion failure is responsible for the occurrence of cracks at the interface in the nanofiber [11]. To improve the mechanical properties of HAp/PLA composite nanofibers for bone tissue engineering, the improvement of interfacial properties between PLA and HA is an important issue to be overcome.

\section{Conclusion}

To disperse HAp in the nanofibers, hot water treatment, oleic acid treatment and stearic acid treatment were conducted. HAp/PLA nanofiber nonwoven fabrics were fabricated by the electrospinning method, and the mechanical properties of HAp/PLA nanofiber nonwoven fabrics were revealed by tensile test. The investigation yielded the following conclusions.

1. HAp particles were dispersed mostly in the PLA nanofiber by surface modification using stearic acid.

2. HAp/PLA nanofiber nonwoven fabrics which contained modified HAp by stearic acid indicated high strength compared to the untreated ones. By dispersing HAp, stress concentration was suppressed.

3. HAp/PLA nanofiber nonwoven fabrics showed lower tensile strength compared to PLA nanofiber nonwoven fabrics. 


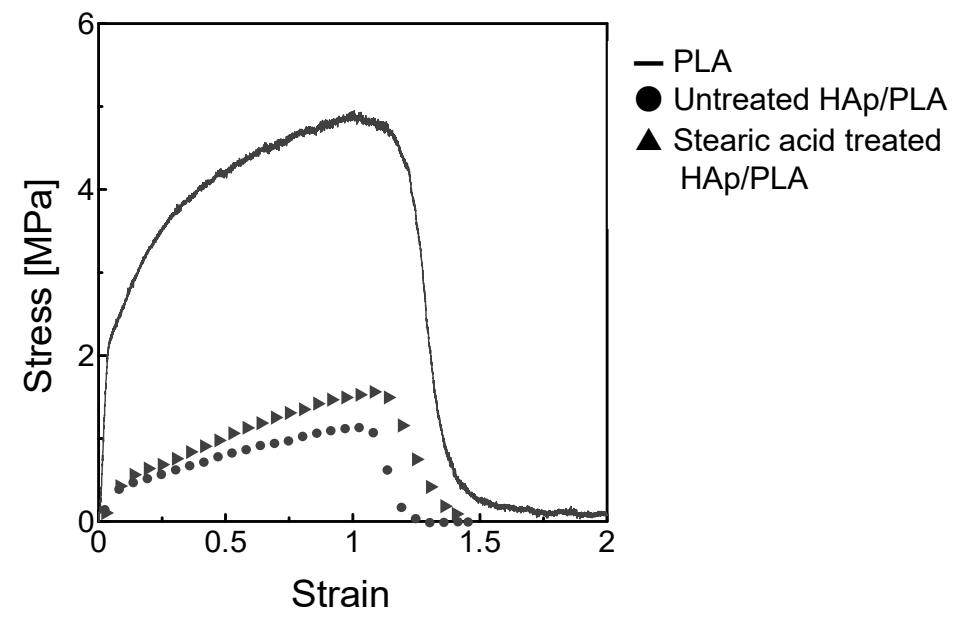

(a) Stress-strain curve

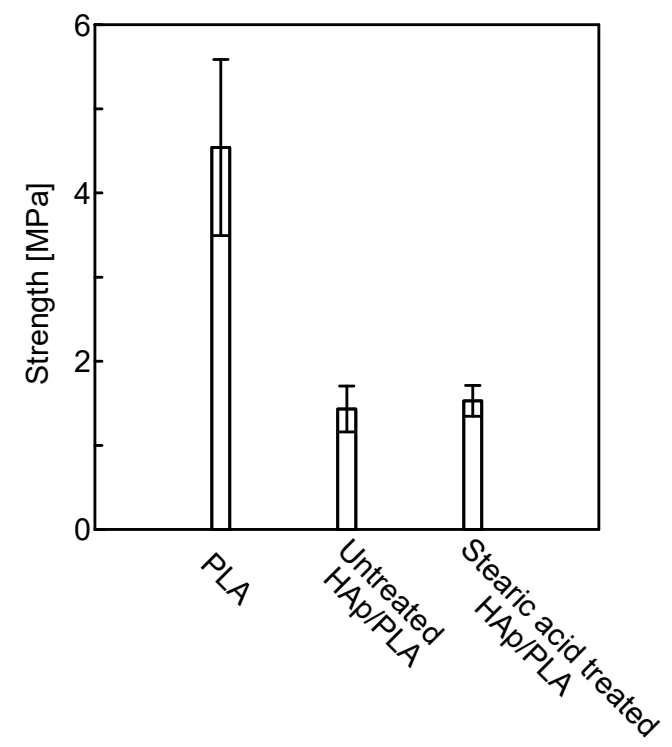

(b) Tensile strength

Figure 8: Results of tensile test for nanofiber nonwoven fabrics. 


\section{References}

[1] Wei, G., Structure and Properties of Nano-Hydroxyapatite/Polymer Composite Scaffolds for Bone Tissue Engineering, Biomaterials 25, pp. 4749-4757, 2004.

[2] Terai, H., A Biodegradable Nanofiber Scaffold by Electrospinning and Its Potential for Bone Tissue Engineering, Biomaterials 24, pp. 2077-2082, 2003.

[3] Drury, L. J., Hydrogels for Tissue Engineering: Scaffold Design Variables and Applications, Biomaterials 24, pp. 4337-4351, 2003.

[4] Lee, H. J., Thermal and Mechanical Characteristics of Poly(l-lactic acid) Nanocomposite Scaffold, Biomaterials 24, pp. 2773-2778, 2003.

[5] Sui, G., Yang, X., "Poly-L-lactic acid/Hydroxyapatite Hybrid Membrane for Bone Tissue Regeneration" Wiley Inter Science, pp. 445-454, 2007.

[6] Huang, Z., Electrospinning and Mechanical Characterization of Gelatin Nanofibers, Polymer 45, pp. 5361-5368, 2004.

[7] Kakuta, M., Science and Technology on Dispersion of Nano-particle, CMC Publication, pp. 295-304, 2006.

[8] Kim, H., Lee, H., Knowles, C. J., Electrospinning Biomedical Nanocomposite Fibers of Hydroxyapaite/Poly(lactic acid) for Bone Regeneration, Journal of Biomedical Materials Research, pp. 643-649, 2006.

[9] Muramoto, S., Dispersing Agent for Fine Ceramics Particles, News of Sanyo Chemicals, Vol. 1-456, p. 81, 2009.

[10] Tanaka, M., Proposal of Novel Interface-control Method in HAp/PLA Composite for Bone Regeneration by Reaction-control Utilizing Photodissociable Protective Groups, The Society of Materials Science, pp. 226-227, 2015.

[11] Sumin, L., Kimura, D., Yokoyama, A., Lee, K., Park, C. J., The Effects of Laundering on the Mechanical Properties of Mass-produced Nanofiber Web for Use in Wear, Textile Research Journal Vol. 79, pp. 1085-1090, 2012. 